\title{
EFFICACY OF CONSERVATIVE TREATMENTS FOR HAND OSTEOARTHRITIS:
}

AN UMBRELLA REVIEW OF INTERVENTION STUDIES

Nicola Veronese, Leonardo Punzi, Lee Smith, Francesco Bolzetta, Alberto Cester, Jacopo Demurtas 


\begin{abstract}
Background: Hand osteoarthritis (OA) is common, but the efficacy/safety of treatment interventions aimed to improve health outcomes in this population are not well understood. Therefore, the aim of this study was to map and grade the effect of interventions for health outcomes in hand OA.

Methods: Umbrella review of systematic reviews with meta-analyses of randomized controlled trials (RCTs) using placebo/no intervention as control group. For outcomes with a $p$-value $<0.05$, the certainty of the evidence was evaluated using the GRADE (Grading of Recommendations Assessment, Development and Evaluation) assessment.
\end{abstract}

Results: From 189 abstracts, 9 meta-analyses (24 outcomes) were included, with eight reporting significant summary results. The use of splints was associated with reduced pain at medium term in thumb carpometacarpal OA (standardized mean difference, $\mathrm{SMD}=-0.70 ; 95 \%$ confidence intervals, $\mathrm{CI}$ : -1.05 to -0.35 ; low certainty), reduced pain in long follow-up RCTs in symptomatic hand OA (SMD=0.80; 95\%CI: $-1.16 ;-0.45 ;$ moderate certainty), and better function (SMD=0.42;95\% $\mathrm{CI}: 0.08 ; 0.70 ;$ low certainty). The use of resistance training $(\mathrm{SMD}=-0.27 ; 95 \% \mathrm{CI}:-0.47 ;-0.07)$ or physical exercise $(\mathrm{SMD}=-$ $0.23 ; 95 \% \mathrm{CI}:-0.42 ;-0.04)$ in improving hand pain and in improving finger joint stiffness $(\mathrm{SMD}=-0.36$; 95\%CI: $-0.58 ;-0.15)$ was supported by a moderate certainty of evidence. The use of intra-articular hyaluronic acid in improving function $(\mathrm{MD}=1.12 ; 95 \% \mathrm{CI}: 0.61 ; 1.64$; moderate certainty of evidence) was the only pharmacological intervention statistically significant.

Conclusion: Only some non-pharmacological interventions are effective in improving health outcomes in hand OA and this evidence is supported by a moderate/low certainty, indicating the necessity of further intervention research.

Key words: hand osteoarthritis; physical activity; splint; randomized controlled trial; umbrella review. 


\section{INTRODUCTION}

Hand osteoarthritis (OA) is a common condition in adults and older people. It is widely known that the presence of hand OA linearly increases with age, with women having rates than men, especially after menopause. [1] In an American study comparing the incidence of different forms of OA in a population living around Boston, the authors, using only radiological information found that the highest incidence was found for knee OA (240/100 000 person-years), with intermediate rates for hand OA (100/100000 person-years) and lowest observed rates for hip OA (88/100000 person-years). [2] These figures were overall confirmed in other surveys, such as in Europe. [3]

Hand OA seems to be associated with several negative outcomes including a high rate of disability [4], poor quality of life [5], whilst the association between hand OA and cardiovascular disease [6] or mortality [7] is less clear. However, hand OA is characterized by a high presence of pain, stiffness and finally limited function making this condition very relevant from a clinical point of view. [8] Despite the clinical importance of hand OA, a few treatments are approved for treating the symptoms (pain, stiffness, poor function) associated with this condition.[9] In 2007, the European League Against Rheumatism (EULAR) proposed some non-pharmacological and pharmacological interventions based on experts' opinion [10], whilst in 2018 other authors updated these recommendations, even if the evidence was mainly based on single randomized controlled trials (RCTs) credibility.[11]

Given this background, we aimed to capture the breadth of outcomes associated with interventions in people affected by hand OA and systematically assess the quality, strength and credibility of these associations. We used the umbrella review methodology to combine evidence from a wide range of outcomes and populations including only RCTs. 


\section{MATERIALS AND METHODS}

\section{Data sources and searches}

We conducted an umbrella review [12], searching MEDLINE, Scopus, Embase databases until $31^{\text {st }}$ December 2019 with: “(Meta-Analysis[ptyp] OR metaanaly*[tiab] OR meta-analy*[tiab] OR Systematic review [ptyp] OR "systematic review" [tiab]) AND (hand osteoarthritis [tiab])". In addition, we handsearched the reference lists of eligible and other relevant articles.

\section{Study selection}

For the aims of this work, we included formal systematic reviews with meta-analyses of intervention studies, which investigated the effect of any kind of interventions (except surgical ones) for the treatment of hand OA. Two authors (JD, NV) performed title and abstract screening, with another independent author (LS) available if needed. Full-texts of all potentially eligible articles were then retrieved by the same two authors and any disagreement was resolved with another independent author (LS).

We included meta-analyses that investigated people affected by hand OA and including RCTs, with at least one group taking placebo or no active intervention, exploring the association of any kind of intervention with any health-related outcome. The type of interventions was consequently categorized as pharmacological and non-pharmacological depending on their nature. Nutritional supplementations were included among the pharmacological interventions. Meta-analyses were included only if they reported study-specific information (i.e. effect size, 95\% confidence intervals [CIs], sample size) or such information could be inferred from the presented data. 


\section{Data extraction}

Two independent investigators (JD, NV), extracted key information for each meta-analysis: first author name; publication year; type of intervention; comparison group; hand OA definition; outcome of interest; follow-up (in months); number of people randomized to active intervention and those randomized to placebo/no intervention. We also extracted the study-specific estimated relative risk for health outcome (mean difference, MD; standardized mean difference, SMD) and 95\% confidence intervals (CIs). We finally extracted the data for the Assessment of Multiple Systematic Reviews (AMSTAR)-2 tool. [13]

When more than one meta-analysis on the same research question was identified, the one with the largest number of participants was selected.

\section{Quality assessment}

We assessed the methodological quality of the included meta-analyses using AMSTAR-2 [13, 14] that ranks the quality of a meta-analysis from critically low to high according to 16 predefined items.

\section{Data synthesis and analysis}

For each meta-analysis, we re-calculated the summary effect size and its $95 \%$ CI by using the randomeffects DerSimonian and Laird. [15] We also estimated the prediction interval (PIs) and its 95\% CI, which further accounts for between-study effects and estimates the certainty of the association if a new study addresses that same association.[16, 17] Between-study inconsistency was estimated with the $I^{2}$ metric, with values $\geq 50 \%$ indicative of high heterogeneity.[18]

We then calculated the evidence of small-study effects (i.e. whether small studies inflated effect sizes). We used the regression asymmetry test [19], using a p-value $<0.10$ with more conservative effects in larger studies as indicative of small-study effects.[20] 
All the analyses were conducted with STATA 13.0 (Stata Corp LP, College station, Texas).

\section{Grading the evidence}

Evidence from meta-analyses of RCTs was assessed in terms of the significance of the summary effect, using a p-value $<0.05$ as the threshold for statistical significance, as recently proposed.[21, 22] For significant outcomes, we evaluated the evidence using the GRADE (Grading of Recommendations, Assessment, Development and Evaluation) assessment.[23] We also considered 95\% PIs (excluding the null or not), small study effects $(\mathrm{P}>0.10)$, and if the largest study was statistically significant or not, as possible indicators of bias in the available evidence. 


\section{RESULTS}

As shown in Figure 1, we identified 189 unique works in three major databases, with 9 meta-analyses (corresponding to 24 different outcomes) finally included in our umbrella review. [24-32]

\section{Meta-analyses of RCTs (vs. placebo/no treatment)}

As reported in Supplementary Table 1, the median number of RCTs meta-analyses for each outcome was only 2 (range 2-6), the median number of participants was 164 (130 to 702). Overall, 18/24 of the interventions were ranked as non-pharmacological, and the most frequent intervention regarded physical exercise ( $n=6$ outcomes) and the use of splints $(n=10)$. Regarding the site of hand OA affected, eight outcomes included all types of hand OA and subtypes, six only symptomatic forms, the other 10 thumbcarpometacarpal, only thumb or trapeziometacarpal forms. Finally, 14 outcomes included evaluation of pain, followed by 8 investigating function as outcome, one handgrip strength and another one stiffness.

Overall, one third of the outcomes included (8/24) reported nominally significant summary results $(p<0.05)$. Table 1 shows the strength of the association between proposed interventions and selected outcomes in people affected by hand OA, using the GRADE. Among seven non-pharmacological interventions, the use of splints was associated with reduced pain sensation at medium term in thumb carpometacarpal $\mathrm{OA}(\mathrm{SMD}=-0.70 ; 95 \% \mathrm{CI}:-1.05$ to -0.35 ; low certainty of evidence $)$, reduced pain in long follow-up RCTs in symptomatic forms of hand OA (SMD=-0.80; 95\%CI: -1.16 ; -0.45 ; moderate certainty of evidence) and a better function ( $\mathrm{SMD}=0.42 ; 95 \% \mathrm{CI}: 0.08 ; 0.70$; low certainty of evidence), in two RCTs for each outcome. The certainty of evidence was mainly given by the small sample sizes included in these RCTs. 
A moderate certainty of evidence supported the use of resistance training ( 5 RCTs, SMD $=-0.27 ; 95 \% \mathrm{CI}$ : $-0.47 ;-0.07)$ or physical exercise $(5 \mathrm{RCTs}, \mathrm{SMD}=-0.23 ; 95 \% \mathrm{CI}:-0.42 ;-0.04)$ in improving hand pain and the use of physical exercise in improving finger joint stiffness ( 4 RCTs, SMD=-0.36; 95\%CI: -0.58 ; -0.15) (Table 1). Conversely, the use of a multimodal intervention was associated with an improvement in pain in trapeziometacarpal OA supported by a very low certainty of evidence.

The only pharmacological intervention associated with a signficant outcome was the use of intra-articular hyaluronic acid in improving function in people affected by thumb OA (MD=1.12; 95\%CI: $0.61 ; 1.64$; moderate certainty of evidence).

Supplementary Table 1 shows other analyses commonly used in the umbrella review methodology. Three outcomes reported a small-study effect, in six outcomes the largest study, in terms of participants, was statistically significant, and only one outcome reported the PIs not including the null value.

As reported in Supplementary Table 2, four meta-analyses were rated "High" according to the AMSTAR-2 criteria, whilst 3 other meta-analyses were rated as "Low" and 2 as "Critically low". 


\section{DISCUSSION}

With this work, we provide a comprehensive overview of the potential pharmacological and nonpharmacological interventions in people affected by hand OA, incorporating evidence from nine metaanalyses. In this regard, we assessed the evidence of RCTs using the GRADE assessment, in order to increase the transparency of these evidences. Overall, we found that, among 24 different interventions investigated, only eight were supported by a statistical significance and of these, five reached a moderate certainty of the evidence. The AMSTAR-2 indicated that works included were accurate in describing risk of bias in studies included in meta-analysis. Moreover, meta-analysis included often presented and followed pre-established protocols and reporting models like PRISMA.

Our umbrella review shows that the large majority of the interventions for hand OA regard nonpharmacological interventions, in particular splints. Using the most common categorization for SMD (i.e. small, moderate, or large effect, if the SMD was $0.2-0.5,0.5-0.7$, and $\geq 0.7$, respectively) [33], we observed that splints are able to significantly reduce pain in thumb carpometacarpal and in symptomatic OA with a large effect, even if this evidence is supported only by a low/moderate certainty of evidence, using the GRADE. From a clinical point of view, it seems that splints might provide a material support

of inflamed joints, finally reducing inflammation with a consequent reduction in pain.[24, 34, 35] A similar effect was suggested for improving function: in our umbrella review, the improvement in function was moderate according to the SMD, but again suffered on the presence of bias and of limited sample size included. Among non-pharmacological interventions the other that we found to be significant is physical exercise.[36] In this case, physical exercise, in particular resistance training, decreased pain with a small effect and, again, the effect of biases and imprecision is unfortunately of importance, even if it is a topic of great importance. Further specific research is needed regarding the importance of physical exercise in improving hand OA outcomes. 
In our umbrella review, the only pharmacological intervention statistically superior to placebo was intraarticular hyaluronic acid in improving function in people specifically affected by thumb OA, in agreement with a robust review regarding this topic.[37] This is somewhat surprising since several medications are commonly used for improving pain and function in hand OA, including topical and oral non-steroidal anti-inflammatory drugs, paracetamol, glucorticoids, intra-articular medications and many others. [11, 38-41] In this regard, the most common guidelines for hand OA $[10,11]$ recognize that very limited research is available for this specific condition regarding medications that are commonly used for types of OA, such as knee OA. [42] Our work further supports the need for high-quality RCTs for hand OA, also due its clinical and epidemiological importance. [8]

The findings of our work should be interpreted within its limitations. First, we used evidence assessment criteria, which were based on already established tools for evaluating the current evidence that can be biased for their nature.[43] Moreover, meta-analyses included studies with relevant differences in design, population and other basic characteristics, that can increase the risk of high heterogeneity. In order to overcome this problem, we used an $\mathrm{I}^{2}<50 \%$ as one of the domains of the GRADE. Second, another common limitation of an umbrella review approach is the use of existing meta-analyses that are related to choices made about what estimates to select from each primary study and how to represent them in the meta-analysis. Third, in this umbrella review a half of the outcomes included only two RCTs and most of the RCTs included small sample sizes strongly limiting our results. It is noteworthy, for example, that only one outcome (i.e. the use of physical exercise in reducing pain) had 95\% PIs excluding the null value.

In conclusion, our umbrella review including nine meta-analyses and 24 different outcomes in people affected by hand OA, found that only a few non-pharmacological interventions are potentially effective 
in improving health outcomes and this evidence is supported by a moderate/low certainty of evidence according to the GRADE. Our work further encourages specific research of high quality RCTs in order to increase the availability of interventions for improving outcomes in people affected by hand OA. 


\section{ACKNOWLEDGEMENTS}

Funding source: None to declare.

Conflict of interest: On behalf of all authors, the corresponding author states that there is no conflict of interest.

Ethical statements: A formal ethical statement was not required, since this is a review of previously published articles. 


\section{FIGURE LEGEND}

\section{Figure 1. PRISMA flow-chart}
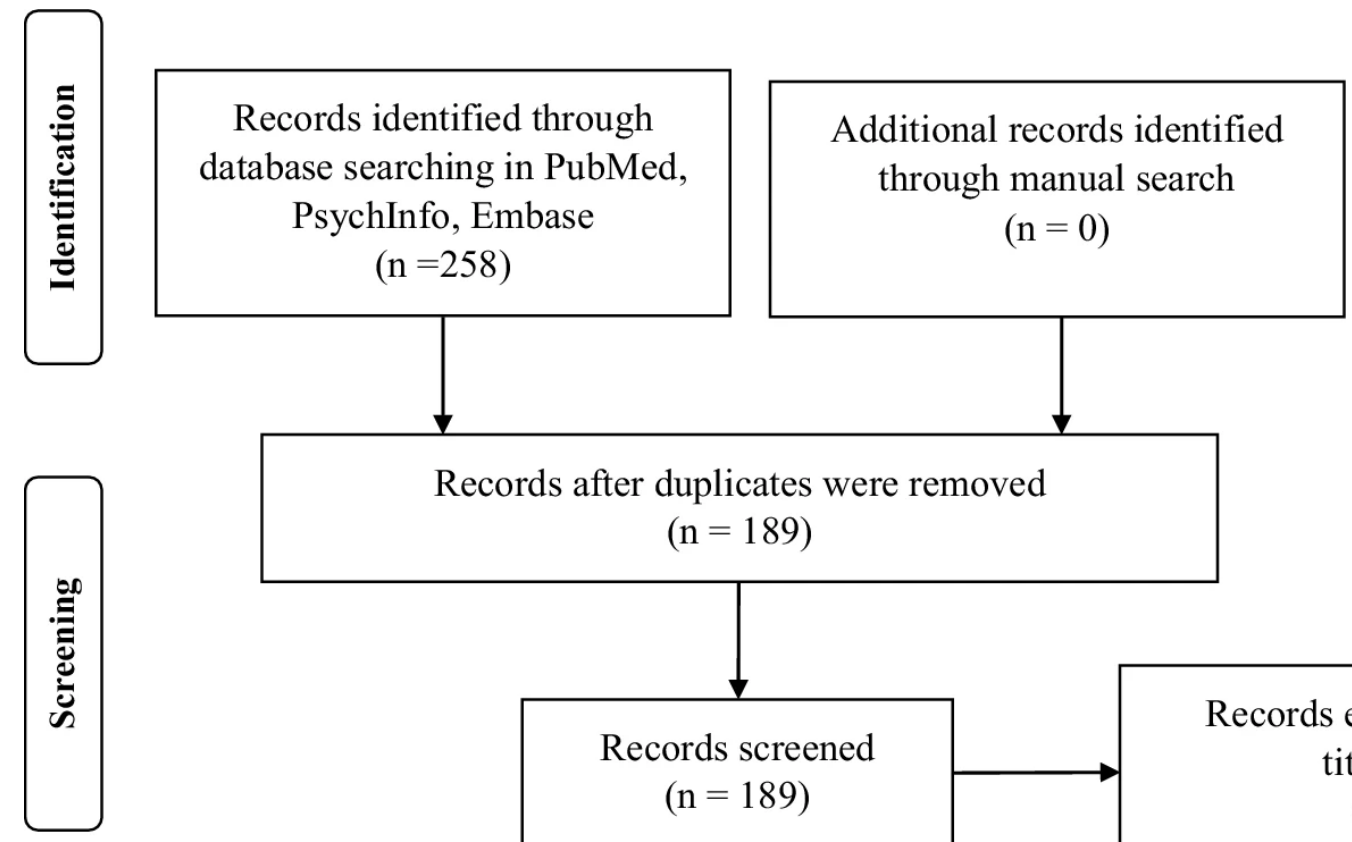

Records after duplicates were removed
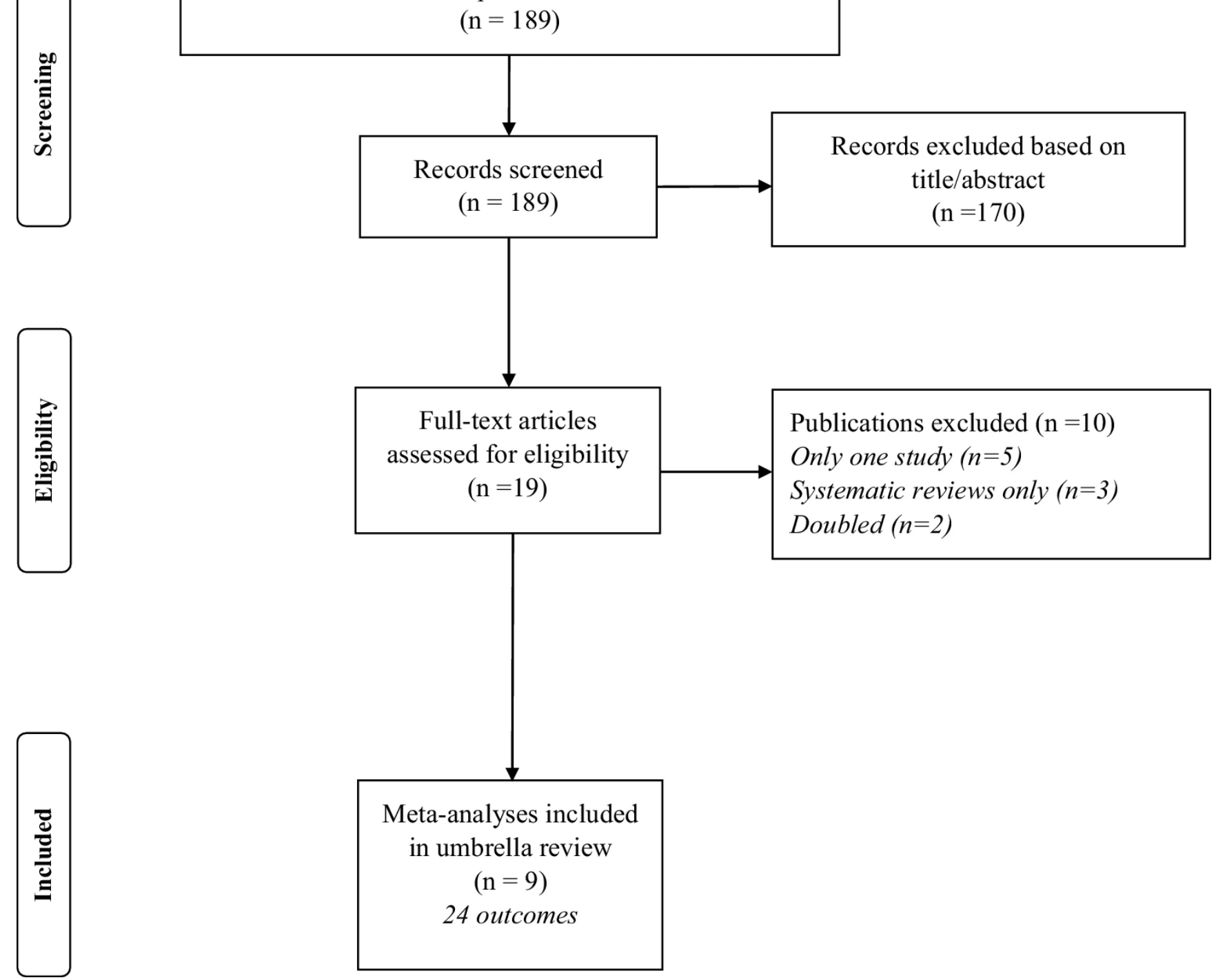


\section{TABLE 1}

Table 1 - GRADE evidence of randomized controlled trials using nonpharmacological and pharmacological interventions for hand osteoarthritis

\begin{tabular}{|c|c|c|c|c|c|c|c|c|c|c|c|}
\hline \multicolumn{7}{|c|}{ Certainty assessment } & \multicolumn{5}{|c|}{ Summary of findings } \\
\hline \multirow{2}{*}{\begin{tabular}{|l} 
No of \\
participants \\
(studies) \\
Follow-up
\end{tabular}} & \multirow[t]{2}{*}{$\begin{array}{l}\text { Risk of } \\
\text { bias }\end{array}$} & \multirow[t]{2}{*}{ Inconsistency } & \multirow[t]{2}{*}{ Indirectness } & \multirow[t]{2}{*}{ Imprecision } & \multirow[t]{2}{*}{ ||Publication bias } & \multirow{2}{*}{$\begin{array}{l}\text { Overall } \\
\text { certainty of } \\
\text { evidence }\end{array}$} & \multicolumn{2}{|c|}{ Study event rates $(\%)$} & \multirow{2}{*}{$\begin{array}{l}\text { Type of intervention (vs. } \\
\text { control group) }\end{array}$} & \multicolumn{2}{|c|}{ Anticipated absolute effects } \\
\hline & & & & & & & $\begin{array}{l}\text { With no } \\
\text { intervention }\end{array}$ & \begin{tabular}{|l} 
With \\
intervention
\end{tabular} & & \begin{tabular}{|l|} 
Risk with no \\
intervention
\end{tabular} & $\begin{array}{l}\text { Risk difference } \\
\text { with intervention }\end{array}$ \\
\hline \multicolumn{12}{|c|}{ Pain (medium follow-up, 3-12 months) in thumb carpometacarpal osteoarthritis } \\
\hline $\begin{array}{l}137 \\
(2 \mathrm{RCTs})\end{array}$ & Serious $^{\mathrm{a}}$ & Not serious & Not serious & Serious ${ }^{\mathrm{b}}$ & None & $\begin{array}{l}\oplus \oplus \bigcirc \bigcirc \\
\text { LOW }\end{array}$ & 65 & 72 & Splints (vs. no splints) & - & \begin{tabular}{|l|} 
SMD -0.70 \\
$(-1.05$ to -0.35$)$
\end{tabular} \\
\hline \multicolumn{12}{|c|}{ Function (medium follow-up, 3-12 months) in thumb carpometacarpal osteoarthritis } \\
\hline $\begin{array}{l}135 \\
(2 \mathrm{RCTs})\end{array}$ & Serious ${ }^{\mathrm{a}}$ & Not serious & Not serious & Serious ${ }^{\mathrm{b}}$ & None & $\begin{array}{l}\oplus \oplus \bigcirc \bigcirc \\
\text { LOW }\end{array}$ & 66 & 69 & Splints (vs. no splints) & - & \begin{tabular}{|l|} 
SMD 0.42 \\
$(0.08-0.77)$
\end{tabular} \\
\hline \multicolumn{12}{|c|}{ Pain (long follow-up) in symptomatic hand osteoarthritis } \\
\hline $\begin{array}{l}152 \\
(2 \mathrm{RCTs})\end{array}$ & $\begin{array}{l}\text { Not } \\
\text { serious }\end{array}$ & Not serious & Not serious ${ }^{\mathrm{b}}$ & Serious $^{\mathrm{b}}$ & None & $\begin{array}{l}\text { } \oplus \oplus \oplus \bigcirc \\
\text { MODERATE }\end{array}$ & 75 & 77 & Splints (vs. no splints) & - & $\begin{array}{l}\text { SMD }-0.80 \\
(-1.16 \text { to }-0.45)\end{array}$ \\
\hline \multicolumn{12}{|l|}{ Hand pain } \\
\hline $\begin{array}{l}381 \\
(5 \mathrm{RCTs})\end{array}$ & Serious ${ }^{\mathrm{c}}$ & Not serious & Not serious & Not serious & None & $\begin{array}{l}\oplus \oplus \oplus \bigcirc \\
\text { MODERATE }\end{array}$ & 193 & 188 & $\begin{array}{l}\text { Resistance training (vs. no } \\
\text { resistance training) }\end{array}$ & - & $\begin{array}{l}\text { SMD }-0.27 \\
(-0.47 \text { to }-0.07)\end{array}$ \\
\hline \multicolumn{12}{|c|}{ Finger joint stiffness } \\
\hline $\begin{array}{l}368 \\
(4 \mathrm{RCTs})\end{array}$ & Serious $^{\mathrm{c}}$ & Not serious & Not serious & Not serious & None & $\begin{array}{l}\oplus \oplus \oplus \bigcirc \\
\text { MODERATE }\end{array}$ & 188 & 180 & $\begin{array}{l}\text { Physical exercise (vs. no } \\
\text { exercise) }\end{array}$ & - & $\begin{array}{l}\text { SMD }-0.36 \\
(-0.58 \text { to }-0.15)\end{array}$ \\
\hline
\end{tabular}




\begin{tabular}{|c|c|c|c|c|c|c|c|c|c|c|c|}
\hline \multicolumn{7}{|c|}{ Certainty assessment } & \multicolumn{5}{|c|}{ Summary of findings } \\
\hline \multirow{2}{*}{\begin{tabular}{|l} 
№ of \\
participants \\
(studies) \\
Follow-up
\end{tabular}} & \multirow[t]{2}{*}{$\begin{array}{l}\text { Risk of } \\
\text { bias }\end{array}$} & \multirow[t]{2}{*}{ Inconsistency } & \multirow[t]{2}{*}{ ||Indirectness } & \multirow[t]{2}{*}{ Imprecision } & \multirow[t]{2}{*}{ Publication bias } & \multirow{2}{*}{$\begin{array}{l}\text { Overall } \\
\text { certainty of } \\
\text { evidence }\end{array}$} & \multicolumn{2}{|c|}{ Study event rates (\%) } & \multirow{2}{*}{$\begin{array}{l}\text { Type of intervention (vs. } \\
\text { control group) }\end{array}$} & \multicolumn{2}{|c|}{ Anticipated absolute effects } \\
\hline & & & & & & & $\begin{array}{l}\text { With no } \\
\text { intervention }\end{array}$ & $\begin{array}{l}\text { With } \\
\text { intervention }\end{array}$ & & $\begin{array}{l}\text { Risk with no } \\
\text { intervention }\end{array}$ & \begin{tabular}{|l|} 
Risk difference \\
with intervention
\end{tabular} \\
\hline $\begin{array}{l}492 \\
(5 \mathrm{RCTs})\end{array}$ & Serious ${ }^{\mathrm{c}}$ & Not serious & Not serious & Not serious & None & $\begin{array}{l}\oplus \oplus \oplus \bigcirc \\
\text { MODERATE }\end{array}$ & 246 & 246 & $\begin{array}{l}\text { Physical exercise (vs. no } \\
\text { exercise) }\end{array}$ & - & $\begin{array}{l}\text { SMD }-0.23 \\
(-0.42 \text { to }-0.04)\end{array}$ \\
\hline \multicolumn{12}{|c|}{ Pain in trapeziometacarpal osteoarthritis } \\
\hline $\begin{array}{l}185 \\
(4 \mathrm{RCTs})\end{array}$ & $\begin{array}{l}\text { Not } \\
\text { serious }\end{array}$ & Very serious ${ }^{\mathrm{d}}$ & Not serious & Serious ${ }^{\mathrm{b}}$ & $\begin{array}{l}\text { Publication bias } \\
\text { strongly } \\
\text { suspected }^{\text {e }}\end{array}$ & $\begin{array}{l}\oplus \bigcirc \bigcirc \bigcirc \\
\text { VERY LOW }\end{array}$ & 91 & 94 & $\begin{array}{l}\text { Physical and occupational } \\
\text { therapy (multimodal) (vs. } \\
\text { no intervention) }\end{array}$ & - & $\begin{array}{l}\mathrm{MD}-3.17 \\
(-5.63 \text { to }-0.71)\end{array}$ \\
\hline \multicolumn{12}{|c|}{ Function in thumb osteoarthritis } \\
\hline $\begin{array}{l}148 \\
(2 \mathrm{RCTs})\end{array}$ & $\begin{array}{l}\text { Not } \\
\text { serious }\end{array}$ & Not serious & Not serious ${ }^{\mathrm{b}}$ & Serious ${ }^{\mathrm{b}}$ & None & $\begin{array}{l}\oplus \oplus \oplus \bigcirc \\
\text { MODERATE }\end{array}$ & 74 & 74 & IAHA (vs. placebo) & - & $\begin{array}{l}\text { MD 1.12 } \\
(0.61-1.64)\end{array}$ \\
\hline
\end{tabular}




\section{REFERENCES}

1. Litwic A, Edwards MH, Dennison EM, Cooper C (2013) Epidemiology and burden of osteoarthritis. British medical bulletin 105:185-199

2. Lawrence RC, Felson DT, Helmick CG, Arnold LM, Choi H, Deyo RA, Gabriel S, Hirsch R, Hochberg MC, Hunder GG (2008) Estimates of the prevalence of arthritis and other rheumatic conditions in the United States: Part II. Arthritis \& Rheumatism 58:26-35

3. Van Saase J, Van Romunde L, Cats A, Vandenbroucke J, Valkenburg H (1989) Epidemiology of osteoarthritis: Zoetermeer survey. Comparison of radiological osteoarthritis in a Dutch population with that in 10 other populations. Annals of the rheumatic diseases 48:271-280

4. Dahaghin S, Bierma-Zeinstra SM, Ginai A, Pols H, Hazes J, Koes B (2005) Prevalence and pattern of radiographic hand osteoarthritis and association with pain and disability (the Rotterdam study). Annals of the rheumatic diseases 64:682-687

5. Slatkowsky-Christensen B, Mowinckel P, Loge JH, Kvien TK (2007) Health-related quality of life in women with symptomatic hand osteoarthritis: a comparison with rheumatoid arthritis patients, healthy controls, and normative data. Arthritis Care \& Research 57:1404-1409

6. Wang H, Bai J, He B, Hu X, Liu D (2016) Osteoarthritis and the risk of cardiovascular disease: a metaanalysis of observational studies. Scientific reports 6:39672

7. Veronese N, Cereda E, Maggi S, Luchini C, Solmi M, Smith T, Denkinger M, Hurley M, Thompson T, Manzato E (2016) Osteoarthritis and mortality: a prospective cohort study and systematic review with metaanalysis. Seminars in arthritis and rheumatism. Elsevier, pp 160-167

8. Reginster J-YL, Arden NK, Haugen IK, Rannou F, Cavalier E, Bruyère O, Branco J, Chapurlat R, Basset SC, Al-Daghri NM (2018) Guidelines for the conduct of pharmacological clinical trials in hand osteoarthritis:

Consensus of a Working Group of the European Society on Clinical and Economic Aspects of Osteoporosis, Osteoarthritis and Musculoskeletal Diseases (ESCEO). Seminars in arthritis and rheumatism. Elsevier, pp 1-8

9. Hermann W, Lambova S, Muller-Ladner U (2018) Current treatment options for osteoarthritis. Current rheumatology reviews 14:108-116

10. Zhang W, Doherty M, Leeb B, Alekseeva L, Arden N, Bijlsma J, Dincer F, Dziedzic K, Hauselmann H, Kaklamanis P (2009) EULAR evidence-based recommendations for the diagnosis of hand osteoarthritis: report of a task force of ESCISIT. Annals of the rheumatic diseases 68:8-17

11. Kroon FP, Carmona L, Schoones JW, Kloppenburg M (2018) Efficacy and safety of non-pharmacological, pharmacological and surgical treatment for hand osteoarthritis: a systematic literature review informing the 2018 update of the EULAR recommendations for the management of hand osteoarthritis. RMD open 4:e000734

12. Ioannidis JP (2009) Integration of evidence from multiple meta-analyses: a primer on umbrella reviews, treatment networks and multiple treatments meta-analyses. CMAJ 181:488-493

13. Shea BJ, Reeves BC, Wells G, Thuku M, Hamel C, Moran J, Moher D, Tugwell P, Welch V, Kristjansson E (2017) AMSTAR 2: a critical appraisal tool for systematic reviews that include randomised or non-randomised studies of healthcare interventions, or both. Bmj 358:j4008

14. Shea BJ, Grimshaw JM, Wells GA, Boers M, Andersson N, Hamel C, Porter AC, Tugwell P, Moher D, Bouter LM (2007) Development of AMSTAR: a measurement tool to assess the methodological quality of systematic reviews. BMC Medical Research Methodology 7:10

15. Lau J, loannidis JP, Schmid CH (1997) Quantitative synthesis in systematic reviews. Ann Intern Med 127:820-826

16. IntHout J, loannidis JPA, Rovers MM, Goeman JJ (2016) Plea for routinely presenting prediction intervals in meta-analysis. BMJ open 6: 
17. Higgins JP, Thompson SG, Spiegelhalter DJ (2009) A re-evaluation of random-effects meta-analysis. J R Stat Soc Ser A Stat Soc 172:137-159

18. Higgins JP, Thompson SG (2002) Quantifying heterogeneity in a meta-analysis. Stat Med 21:1539-1558

19. Egger M, Davey Smith G, Schneider M, Minder C (1997) Bias in meta-analysis detected by a simple, graphical test. BMJ 315:629-634

20. Carvalho AF, Kohler CA, Brunoni AR, Miskowiak KW, Herrmann N, Lanctot KL, Hyphantis TN, Quevedo J, Fernandes BS, Berk M (2016) Bias in Peripheral Depression Biomarkers. Psychotherapy and psychosomatics 85:81-90

21. Ioannidis JA (2018) The proposal to lower $p$ value thresholds to .005. Jama 319:1429-1430

22. Benjamin DJ, Berger JO, Johannesson M, et al. (2018) Redefine statistical significance. Nature Human Behaviour 2:6-10

23. Guyatt GH, Oxman AD, Vist GE, Kunz R, Falck-Ytter Y, Alonso-Coello P, Schünemann HJ (2008) GRADE: an emerging consensus on rating quality of evidence and strength of recommendations. Bmj 336:924

24. Kjeken I, Smedslund G, Moe RH, Slatkowsky-Christensen B, Uhlig T, Hagen KB (2011) Systematic review of design and effects of splints and exercise programs in hand osteoarthritis. Arthritis Care Res (Hoboken) 63:834-848

25. Buhler M, Chapple CM, Stebbings S, Sangelaji B, Baxter GD (2019) Effectiveness of splinting for pain and function in people with thumb carpometacarpal osteoarthritis: a systematic review with meta-analysis. Osteoarthritis Cartilage 27:547-559

26. Persson MSM, Stocks J, Walsh DA, Doherty M, Zhang W (2018) The relative efficacy of topical nonsteroidal anti-inflammatory drugs and capsaicin in osteoarthritis: a network meta-analysis of randomised controlled trials. Osteoarthritis Cartilage 26:1575-1582

27. Persson MSM, Sarmanova A, Doherty M, Zhang W (2018) Conventional and biologic disease-modifying anti-rheumatic drugs for osteoarthritis: a meta-analysis of randomized controlled trials. Rheumatology

57:1830-1837

28. Trellu S, Dadoun S, Berenbaum F, Fautrel B, Gossec L (2015) Intra-articular injections in thumb osteoarthritis: A systematic review and meta-analysis of randomized controlled trials. Joint, bone, spine : revue du rhumatisme 82:315-319

29. Osteras N, Kjeken I, Smedslund G, Moe RH, Slatkowsky-Christensen B, Uhlig T, Hagen KB (2017) Exercise for Hand Osteoarthritis: A Cochrane Systematic Review. J Rheumatol 44:1850-1858

30. Magni NE, McNair PJ, Rice DA (2017) The effects of resistance training on muscle strength, joint pain, and hand function in individuals with hand osteoarthritis: a systematic review and meta-analysis. Arthritis Res Ther 19:131

31. Aebischer B, Elsig S, Taeymans J (2016) Effectiveness of physical and occupational therapy on pain, function and quality of life in patients with trapeziometacarpal osteoarthritis - A systematic review and metaanalysis. Hand therapy 21:5-15

32. Kroon FP, Rubio R, Schoones JW, Kloppenburg M (2016) Intra-Articular Therapies in the Treatment of Hand Osteoarthritis: A Systematic Literature Review. Drugs Aging 33:119-133

33. Cohen J (1988) The t test for means. Statistical power analysis for the behavioural sciences. Hillsdale, NJ: Lawrence Erlbaum Associates,

34. Hamasaki T, Laprise S, Harris PG, Bureau NJ, Gaudreault N, Ziegler D, Choinière M (2019) Efficacy of non-surgical interventions for trapeziometacarpal (thumb base) osteoarthritis: A systematic review. Arthritis care \& research

35. Baradaran A, Baradaran A, Ebrahimzadeh MH, Kachooei AR, Rivlin M, Beredjiklian P (2018) Comparison of custom-made versus prefabricated thumb splinting for carpometacarpal arthrosis: A systematic review and meta-analysis. Archives of Bone and Joint Surgery 6:478

36. Nguyen C, Lefevre-Colau M-M, Poiraudeau S, Rannou F (2016) Rehabilitation (exercise and strength training) and osteoarthritis: A critical narrative review. Annals of physical and rehabilitation medicine 59:190195 
37. Tran K, Loshak H (2019) Intra-Articular Hyaluronic Acid for Viscosupplementation in Osteoarthritis of the Hand, Shoulder, and Temporomandibular Joint: A Review of Clinical Effectiveness and Safety.

38. Guedes V, Castro JP, Brito I (2018) Topical capsaicin for pain in osteoarthritis: A literature review. Reumatología Clínica (English Edition) 14:40-45

39. Fowler A, Swindells M, Burke F (2015) Intra-articular corticosteroid injections to manage trapeziometacarpal osteoarthritis-a systematic review. Hand 10:583-592

40. Urits I, Smoots D, Franscioni H, Patel A, Fackler N, Wiley S, Berger AA, Kassem H, Urman RD, Manchikanti L (2020) Injection Techniques for Common Chronic Pain Conditions of the Foot: A Comprehensive Review. Pain and therapy 1-16

41. Revel FB, Fayet M, Hagen M (2020) Topical Diclofenac, an Efficacious Treatment for Osteoarthritis: A Narrative Review. Rheumatology and therapy 1-20

42. Bruyère $\mathrm{O}$, Honvo $\mathrm{G}$, Veronese $\mathrm{N}$, Arden NK, Branco J, Curtis EM, Al-Daghri NM, Herrero-Beaumont $\mathrm{G}$, Martel-Pelletier J, Pelletier JP (2019) An updated algorithm recommendation for the management of knee osteoarthritis from the European Society for Clinical and Economic Aspects of Osteoporosis, Osteoarthritis and Musculoskeletal Diseases (ESCEO). Seminars in arthritis and rheumatism

43. Li X, Meng X, Timofeeva M, Tzoulaki I, Tsilidis KK, Ioannidis PA, Campbell H, Theodoratou E (2017) Serum uric acid levels and multiple health outcomes: umbrella review of evidence from observational studies, randomised controlled trials, and Mendelian randomisation studies. Bmj 357: 


\section{SUPPLEMENTARY INFORMATION}

\section{Supplementary Table 1. Descriptive characteristics (and additional analyses) for the outcomes}

\section{included}

\begin{tabular}{|c|c|c|c|c|c|c|c|c|c|c|c|}
\hline Intervention & $\begin{array}{c}\text { Comparison } \\
\text { group }\end{array}$ & $\begin{array}{l}\text { Hand OA } \\
\text { definition }\end{array}$ & Outcome & $\begin{array}{c}\text { Number } \\
\text { of } \\
\text { studies }\end{array}$ & Cases & Controls & $\begin{array}{l}\text { Sample } \\
\text { size }\end{array}$ & $\begin{array}{l}\text { Type of } \\
\text { metric }\end{array}$ & Mean ES (RE) & $\mathbf{P}$ & $\mathbf{I}^{2}$ \\
\hline $\mathrm{CS}$ & placebo & thumb & pain & 2 & 82 & 82 & 164 & MD & $\begin{array}{c}-1.12 \\
(-3.69 ; 1.29)\end{array}$ & 0.35 & 96.6 \\
\hline DMARDS & placebo & all types & pain & 6 & 347 & 355 & 702 & ES & $\begin{array}{c}0.1 \\
(-0.05 ; 0.24)\end{array}$ & 0.19 & 0 \\
\hline exercise & no exercise & all types & pain & 4 & 188 & 193 & 381 & SMD & $\begin{array}{c}-0.27 \\
(-0.47 ;-0.07)\end{array}$ & 0.00005 & 7.2 \\
\hline exercise & no exercise & all types & stiffness & 4 & 180 & 188 & 368 & SMD & $\begin{array}{c}-0.36 \\
(-0.58 ;-0.15)\end{array}$ & 0.001 & 7.6 \\
\hline exercise & no exercise & all types & function & 4 & 181 & 188 & 369 & SMD & $\begin{array}{c}-0.28 \\
(-0.59 ; 0.02)\end{array}$ & 0.06 & 51 \\
\hline hyaluronic & placebo & thumb & function & 2 & 74 & 74 & 148 & MD & $\begin{array}{c}-1.12 \\
(-1.64 ;-0.61)\end{array}$ & 0.00002 & 26.5 \\
\hline hyaluronic & placebo & thumb & pain & 2 & 74 & 74 & 148 & MD & $\begin{array}{c}-0.95 \\
(-3.78 ; 1.97)\end{array}$ & 0.52 & 96 \\
\hline $\begin{array}{l}\text { intra- } \\
\text { articular CS }\end{array}$ & placebo & thumb-base & pain & 2 & 85 & 81 & 166 & MD & $\begin{array}{c}-3.56 \\
(-13.9 ; 6.75)\end{array}$ & 0.5 & 50.7 \\
\hline $\begin{array}{l}\text { physical and } \\
\text { occupational } \\
\text { therapy } \\
\text { (multimodal) }\end{array}$ & none & trapeziometacarpal & pain & 4 & 94 & 91 & 185 & MD & $\begin{array}{c}-3.17 \\
(-5.63 ;-0.71)\end{array}$ & 0.01 & 97.3 \\
\hline $\begin{array}{l}\text { physical and } \\
\text { occupational } \\
\text { therapy } \\
\text { (multimodal) }\end{array}$ & none & trapeziometacarpal & function & 3 & 65 & 65 & 130 & SMD & $\begin{array}{c}-0.66 \\
(-1.55 ; 0.23)\end{array}$ & 0.15 & 82.9 \\
\hline $\begin{array}{l}\text { resistance } \\
\text { training }\end{array}$ & no resistance training & all types & pain & 5 & 246 & 246 & 492 & SMD & $\begin{array}{c}-0.23 \\
(-0.42 ;-0.04)\end{array}$ & 0.02 & 0 \\
\hline $\begin{array}{l}\text { resistance } \\
\text { training }\end{array}$ & no resistance training & all types & $\begin{array}{c}\text { grip } \\
\text { strength }\end{array}$ & 4 & 246 & 246 & 492 & MD & $\begin{array}{c}1.35 \\
(-0.84 ; 3.53)\end{array}$ & 0.23 & 49.9 \\
\hline $\begin{array}{l}\text { resistance } \\
\text { training }\end{array}$ & no resistance training & all types & function & 4 & 246 & 246 & 492 & SMD & $\begin{array}{c}-0.10 \\
(-0.33 ; 0.13)\end{array}$ & 0.39 & 27.4 \\
\hline splints & no splints & symptomatic & $\begin{array}{c}\text { pain long } \\
\mathrm{fu}\end{array}$ & 2 & 77 & 75 & 152 & SMD & $\begin{array}{c}-0.80 \\
(-1.16 ;-0.45)\end{array}$ & 7.17E-06 & 0 \\
\hline splints & no splints & $\begin{array}{c}\text { thumb } \\
\text { carpometacarpal }\end{array}$ & $\begin{array}{c}\text { pain } \\
\text { medium } \\
\text { fu }\end{array}$ & 2 & 72 & 65 & 137 & SMD & $\begin{array}{c}-0.70 \\
(-1.05 ;-0.35)\end{array}$ & $8.00 \mathrm{E}-05$ & 0 \\
\hline splints & no splints & $\begin{array}{c}\text { thumb } \\
\text { carpometacarpal }\end{array}$ & $\begin{array}{l}\text { function } \\
\text { medium } \\
\text { fu }\end{array}$ & 2 & 69 & 66 & 135 & SMD & $\begin{array}{c}-0.42 \\
(-0.77 ;-0.08)\end{array}$ & 0.02 & 0 \\
\hline splints & no splints & symptomatic & $\begin{array}{l}\text { pinch } \\
\text { short fu }\end{array}$ & 2 & 77 & 75 & 152 & SMD & $\begin{array}{c}0.26 \\
(-0.08 ; 0.59)\end{array}$ & 0.13 & 0 \\
\hline splints & no splints & symptomatic & $\begin{array}{l}\text { function } \\
\text { short fu }\end{array}$ & 2 & 77 & 75 & 152 & SMD & $\begin{array}{c}0.25 \\
(-0.08 ; 0.58)\end{array}$ & 0.14 & 0 \\
\hline splints & no splints & symptomatic & $\begin{array}{l}\text { pinch } \\
\text { long fu }\end{array}$ & 2 & 77 & 75 & 152 & SMD & $\begin{array}{c}0.23 \\
(-0.10 ; 0.57)\end{array}$ & 0.17 & 0 \\
\hline splints & no splints & symptomatic & $\begin{array}{l}\text { function } \\
\text { long fu }\end{array}$ & 2 & 77 & 75 & 152 & SMD & $\begin{array}{c}-0.22 \\
(-0.56 ; 0.12)\end{array}$ & 0.2 & 0 \\
\hline splints & no splints & $\begin{array}{c}\text { thumb } \\
\text { carpometacarpal }\end{array}$ & $\begin{array}{l}\text { pain } \\
\text { short fu }\end{array}$ & 4 & 119 & 102 & 221 & SMD & $\begin{array}{c}-0.23 \\
(-0.59 ; 0.13)\end{array}$ & 0.2 & 38.1 \\
\hline splints & no splints & symptomatic & $\begin{array}{c}\text { pain } \\
\text { short fu }\end{array}$ & 2 & 77 & 75 & 152 & SMD & $\begin{array}{c}0.63 \\
(-0.51 ; 1.78)\end{array}$ & 0.28 & 88.3 \\
\hline splints & no splints & $\begin{array}{c}\text { thumb } \\
\text { carpometacarpal }\end{array}$ & $\begin{array}{l}\text { function } \\
\text { short fu }\end{array}$ & 4 & 118 & 103 & 221 & SMD & $\begin{array}{c}0.12 \\
(-0.15 ; 0.38)\end{array}$ & 0.39 & 0 \\
\hline $\begin{array}{c}\text { topical } \\
\text { NSAIDS }\end{array}$ & placebo & all types & pain & 2 & 218 & 207 & 425 & SMD & $\begin{array}{c}0.48 \\
(-0.83 ; 1.80)\end{array}$ & 0.47 & 0 \\
\hline
\end{tabular}




\section{Abbreviations:}

CI: Confidence interval; SMD: Standardised mean difference; MD: Mean difference; ES: Effect size; NA: not available; OA: osteoarthritis; NSAIDS: non steroidal anti-inflamatory drugs; DMARDS: disease modifying anti-rheumatoid drugs. 
Supplementary Table 2. AMSTAR 2 quality assessment of meta-analyses of RCTs

\begin{tabular}{|c|c|c|c|c|c|c|c|c|c|c|c|c|c|c|c|c|c|}
\hline & \multicolumn{17}{|c|}{ AMSTAR 2 items ${ }^{\mathrm{a}, \mathrm{c}}$} \\
\hline $\begin{array}{l}\text { Author, Year } \\
\text { [Reference] }\end{array}$ & 1 & $2^{b}$ & 3 & $4^{b}$ & 5 & 6 & $7^{b}$ & 8 & $9^{b}$ & 10 & $\begin{array}{c}11 \\
b\end{array}$ & 12 & $\begin{array}{c}13 \\
\text { b }\end{array}$ & 14 & $\begin{array}{c}15 \\
\mathrm{~b}\end{array}$ & 16 & $\begin{array}{l}\text { Overall rating } \\
\text { (based on } \\
\text { critical } \\
\text { domains) }^{\mathbf{d}}\end{array}$ \\
\hline Østeras 2019 & $\bar{Y}$ & $\mathrm{Y}$ & $\mathrm{Y}$ & $\mathrm{Y}$ & $\bar{Y}$ & $\bar{Y}$ & $\mathrm{Y}$ & $\bar{Y}$ & $\mathrm{Y}$ & $\mathrm{Y}$ & $\mathrm{Y}$ & $\bar{Y}$ & $\mathrm{Y}$ & $\mathrm{Y}$ & $\mathrm{Y}$ & $\mathrm{Y}$ & High \\
\hline Magni 2017 & $\bar{Y}$ & $\mathrm{~N}$ & $\mathrm{Y}$ & $\mathrm{Y}$ & $\bar{Y}$ & $\bar{Y}$ & $\mathrm{Y}$ & $\mathrm{Y}$ & $\mathrm{Y}$ & $\mathrm{Y}$ & $\mathrm{Y}$ & $\overline{\mathrm{Y}}$ & $\mathrm{Y}$ & $\bar{Y}$ & $\mathrm{Y}$ & $\mathrm{Y}$ & Low \\
\hline Aebischer 2016 & $\mathrm{Y}$ & $\mathrm{Y}$ & $\mathrm{Y}$ & $\mathrm{Y}$ & $\mathrm{Y}$ & $\mathrm{Y}$ & $\mathrm{Y}$ & $\mathrm{Y}$ & $\mathrm{Y}$ & $\mathrm{N}$ & $\mathrm{Y}$ & $\mathrm{Y}$ & Y & $\mathrm{Y}$ & $\mathrm{N}$ & $\mathrm{Y}$ & Low \\
\hline Kroon 2016 & $\mathrm{Y}$ & $\mathrm{N}$ & $\mathrm{Y}$ & $\mathrm{Y}$ & $\mathrm{Y}$ & $\mathrm{Y}$ & $\mathrm{Y}$ & $\mathrm{Y}$ & $\mathrm{Y}$ & $\mathrm{Y}$ & $\mathrm{Y}$ & $\mathrm{Y}$ & $\mathrm{Y}$ & $\mathrm{Y}$ & $\mathrm{Y}$ & $\mathrm{Y}$ & Low \\
\hline Kjeken 2011 & $\mathrm{Y}$ & $\mathrm{N}$ & $\mathrm{Y}$ & $\mathrm{Y}$ & $\mathrm{Y}$ & $\mathrm{Y}$ & $\mathrm{N}$ & $\mathrm{Y}$ & $\mathrm{Y}$ & $\mathrm{N}$ & $\mathrm{Y}$ & $\mathrm{N}$ & $\mathrm{N}$ & $\mathrm{Y}$ & Y & $\mathrm{Y}$ & Critically low \\
\hline Buhler 2019 & $\mathrm{Y}$ & $\mathrm{Y}$ & $\mathrm{Y}$ & $\mathrm{Y}$ & $\mathrm{Y}$ & $\mathrm{Y}$ & $\mathrm{Y}$ & $\mathrm{Y}$ & $\mathrm{Y}$ & $\mathrm{Y}$ & $\mathrm{Y}$ & $\mathrm{Y}$ & $\mathrm{Y}$ & $\mathrm{Y}$ & $\mathrm{Y}$ & $\mathrm{Y}$ & High \\
\hline Persson 2018 & $\mathrm{Y}$ & $\mathrm{Y}$ & $\mathrm{Y}$ & $\mathrm{Y}$ & $\mathrm{Y}$ & $\mathrm{Y}$ & $\mathrm{Y}$ & $\mathrm{Y}$ & Y & $\mathrm{Y}$ & $\mathrm{Y}$ & $\mathrm{Y}$ & $\mathrm{Y}$ & $\mathrm{Y}$ & $\mathrm{Y}$ & $\mathrm{Y}$ & High \\
\hline Persson 2018 & $\mathrm{Y}$ & $\mathrm{Y}$ & $\mathrm{Y}$ & $\mathrm{Y}$ & $\mathrm{Y}$ & $\mathrm{Y}$ & $\mathrm{Y}$ & $\mathrm{Y}$ & $\mathrm{Y}$ & $\mathrm{Y}$ & $\mathrm{Y}$ & $\mathrm{Y}$ & $\mathrm{Y}$ & $\mathrm{Y}$ & $\mathrm{Y}$ & $\mathrm{Y}$ & High \\
\hline Trellu 2015 & $\mathrm{Y}$ & $\mathrm{N}$ & $\mathrm{Y}$ & $\mathrm{Y}$ & $\mathrm{Y}$ & $\mathrm{Y}$ & $\mathrm{Y}$ & $\mathrm{Y}$ & $\mathrm{Y}$ & $\mathrm{N}$ & $\mathrm{Y}$ & $\mathrm{Y}$ & $\mathrm{N}$ & $\mathrm{Y}$ & Y & $\mathrm{Y}$ & Critically low \\
\hline
\end{tabular}

${ }^{a}$ Yes, No, Other

${ }^{\mathrm{b}}$ Critical Domains

${ }^{c}$ AMSTAR 2 items:

1. Did the research questions and inclusion criteria for the review include the components of PICO (Population, Intervention, Comparator group, Outcome)? YES/NO. For yes, must have all four.

2. Did the report of the review contain an explicit statement that the review methods were established prior to the conduct of the review and did the report justify any significant deviations from the protocol? YES, PARTIAL YES, NO. For Partial YES: the authors state that they had a written protocol or guide that included ALL the following (review question(s), a search strategy, inclusion/exclusion criteria, a risk of bias assessment). For YES: as for partial yes, plus the protocol should be registered and should also have specified: a meta-analysis/synthesis plan, if appropriate, and a plan for investigating causes of heterogeneity, justification for any deviations from the protocol.

3. Did the review authors explain their selection of the study designs for inclusion in the review? YES/NO. For YES, the review should satisfy one of the following: explanation for including only RCTs, or explanation for including only NRSI, or explanation for including both RCTs and NRSI.

4. Did the review authors use a comprehensive literature search strategy? YES, PARTIAL YES, NO. for PARTIAL YES must have all of the following: searched at least 2 databases (relevant to research question), provided key word and/or search strategy, justified publication restrictions (eg. Language). For YES should also have all of the following: searched the reference lists/biographies of included studies, searched trial/study registries, included/consulted content experts in the field, searched for grey literature where relevant, conducted search within 24 months of completion of the review.

5. Did the review authors perform study selection in duplicate? YES/NO. for YES, either ONE of the following: at least two reviewers independently agreed on selection of eligible studies and achieved consensus on which studies to include OR two reviewers selected a sample of eligible studies and achieved good agreement (at least 80 per cent) with the remainder selected by one reviewer.

6. Did the review authors perform data extraction in duplicate? YES/NO. For YES, either one of the following: at least two reviewers achieved consensus on which data to extract from included studies OR two reviewers extracted data from a sample of eligible studies and achieved good agreement (at least 80 per cent) with the remainder extracted by one reviewer. 
7. Did the review authors provide a list of excluded studies to justify the exclusions? YES, PARTIAL YES, NO. FOR partial yes must provide a list of all potentially relevant studies that were read in full text form but excluded from the review. For YES must also have justified the exclusion from the review of each potentially relevant study.

8. Did the review authors describe the included studies in adequate detail? YES, PARTIAL YES, NO. For PARTIAL YES, must describe all of the following: populations, interventions, comparators, outcomes, research designs. For YES should also have all of the following: described populations in detail, described intervention and comparator in detail (including doses where relevant), described study setting, timeframe or follow-up.

9. Did the review authors use a satisfactory technique for assessing the risk of bias (RoB) in individual studies that were included in the review? For RCTs: YES, PARTIAL YES, NO, INCLUDES ONLY NRSI. For PARTIAL YES must have assessed RoB from unconcealed allocation and lack of blinding of patients and assessors when assessing outcomes (unnecessary for objective outcomes such as all cause mortality); for YES must also have assessed RoB from allocation sequence that was not truly random and selection of the reported result from among multiple measurements or analyses of a specified outcome. For NRSI (Non Randomized Studies of Intervention): YES, PARTIAL YES, NO, INCLUDES ONLY RCTs. For PARTIAL YES must have assessed RoB from confounding and from selection bias. For YES, must also have assessed methods used to ascertain exposures and outcomes, and selection of the reported results from among multiple measurements or analyses of a specified outcome.

10. Did the review authors report on the sources of funding for the studies included in the review? YES/NO. For YES: must have reported on the sources of funding for individual studies included in the review. Note: reporting that the reviewers looked for this information but it was not reported by study authors also qualifies

11. If meta-analysis was performed, did the review authors use appropriate methods for statistical combination of results? For RCTs: YES, NO, NO META-ANALYSIS. For YES: the authors justified combining the data in a metaanalysis and they used an appropriate weighted technique to combine study results and adjusted for heterogeneity if present and investigated the causes of heterogeneity. For NRSI: YES, NO, NO META-ANALYSIS CONDUCTED. For YES: the authors justified combining the data in a meta-analysis and they used an appropriate weighted technique to combine study results, adjusting for heterogeneity if present, and they statistically combined effects estimates from NRSI that were adjusted for confounding, rather than combining raw data, or justified combining raw data when adjusted effect estimates were not available, and they reported separate summary estimates for RCTs and NRSI separately when both were included in the review.

12. If meta-analysis was performed, did the review authors assess the potential impact of RoB in individual studies on the results of the meta-analysis or other evidence synthesis? YES, NO, NO META-ANALYSIS INCLUDED. For YES: included only low risk of bias RCTs or, if the pooled estimate was based on RCTs and/or NRSI at variable RoB, the authors performed analysis ton investigate possible impact of RoB on summary estimates of effect.

13. Did the review authors account for $R o B$ in individual studies when interpreting/discussing the results of the review? YES/NO. for YES: included only low risk of bias RCTs or, if RCTs with moderate or high RoB, or NRSI were included, the review provided a discussion of the key impact of RoB on the results

14. Did the review authors provide a satisfactory explanation for, and discussion of, any heterogeneity observed in the results of the review? YES/NO. For Yes: there was no significant heterogeneity in the results OR if heterogeneity was present the authors performed an investigation of sources of any heterogeneity in the results and discussed the impact of this on the results of the review

15. If they performed quantitative synthesis did the review authors carry out an adequate investigation of publication bias (small study bias) and discuss its likely impact on the results of the review? YES, NO, NO METAANALYSIS CONDUCTED. For YES: performed graphical statistical tests for publication bias and discussed the likelihood and magnitude of impact of publication bias

16. Did the review authors report any potential sources of conflict of interest, including any funding they received for conducting the review? YES/NO. For Yes: the authors reported no competing interests OR the authors described their funding sources and how they managed potential conflicts of interest.

${ }^{d}$ Rating overall confidence in the results of the review:

HIGH: no on one non-critical weakness: the systematic review provides an accurate and comprehensive summary of the results of the available studies that address the question of interest

MODERATE: more than one non critical weakness (multiple non-critical weaknesses may diminish confidence in the review and it may be appropriate to move the overall appraisal down from moderate to low confidence): the systematic review has more than one weakness but no critical flaws. It may provide an accurate summary of the results of the available studies that were included in the review 
LOW: one critical flaw with or without non-critical weaknesses: the review has a critical flaw and may not provide an accurate and comprehensive summary of the available studies that address the question of interest

CRITICALLY LOW: more than one critical flaw with or without non-critical weaknesses: the review has more than one critical flaw and should not be relied on to provide an accurate and comprehensive summary of the available studies 